\title{
Uniqueness of Positive Radial Solutions for a Class of Semipositone Systems on the Exterior of a Ball
}

\author{
Alhussein Mohamed ${ }^{*}$, Khalid Ahmed Abbakar ${ }^{1,2}$, Abuzar Awad', Omer Khalil1,3, \\ Bechir Mahamat Acyl1, Abdoulaye Ali Youssouf ${ }^{1}$, Mohammed Mousa1 \\ ${ }^{1}$ College of Mathematics and Statistics, Northwest Normal University, Lanzhou, China \\ ${ }^{2}$ Department of Mathematics and Physics, Faculty of Education, University of Gadarif, Gadarif, Sudan \\ ${ }^{3}$ Department of Science, College of Education, Sudan University of Science and Technology, Khartoum, Sudan \\ Email: *tethussain09@outlook.com
}

How to cite this paper: Mohamed, A., Abbakar, K.A., Awad, A., Khalil, O., Acyl, B.M., Youssouf, A.A. and Mousa, M. (2021) Uniqueness of Positive Radial Solutions for a Class of Semipositone Systems on the Exterior of a Ball. Applied Mathematics, 12, 131-146.

https://doi.org/10.4236/am.2021.123009

Received: November 16, 2020

Accepted: March 9, 2021

Published: March 12, 2021

Copyright $\odot 2021$ by author(s) and Scientific Research Publishing Inc. This work is licensed under the Creative Commons Attribution International License (CC BY 4.0).

http://creativecommons.org/licenses/by/4.0/ (c) (i) Open Access

\section{Abstract}

In this paper, we study the positive radial solutions for elliptic systems to the

nonlinear BVP:

$$
\left\{\begin{array}{l}
-\Delta u=\lambda k_{1}(|x|) f_{1}(u, v) \text { on } \Omega, \\
-\Delta v=\lambda k_{2}(|x|) f_{2}(u, v) \text { on } \Omega, \\
u(x)=v(x)=0 \quad \text { on }|x| \rightarrow \infty, \\
\frac{\partial u}{\partial \eta}+\tilde{c}_{1}(u) u=0 \quad \text { on }|x|=r_{0}, \quad \text {, where } \Delta u=\operatorname{div}(\nabla u) \text { and } \\
\frac{\partial v}{\partial \eta}+\tilde{c}_{2}(v) v=0, \quad \text { on }|x|=r_{0} .
\end{array}\right.
$$

$\Delta v=\operatorname{div}(\nabla v)$ are the Laplacian of $u, \lambda$ is a positive parameter,

$\Omega=\left\{x \in \mathbb{R}^{n}: N>2,|x|>r_{0}, r_{0}>0\right\}$, let $i=[1,2]$ then $K_{i}:\left[r_{0}, \infty\right) \rightarrow(0, \infty)$ is a continuous function such that $\lim _{r \rightarrow \infty} k_{i}(r)=0$ and $\frac{\partial}{\partial \eta}$ is The external natural derivative, and $\tilde{c}_{i}:[0, \infty) \rightarrow(0, \infty)$ is a continuous function. We discuss existence and multiplicity results for classes of $f$ with a) $f_{i}>0, \mathrm{~b}$ ) $f_{i}<0$, and c) $f_{i}=0$. We base our presence and multiple outcomes via the Sub-solutions method. We also discuss some unique findings.

\section{Keywords}

Elliptic System, Positive Radial Solution, Exterior Domains, Fixed Point Index

\section{Introduction}

In reaction diffusion processes, steady states define the long term dynamics. 
Here we consider a steady state reaction diffusion equation on an exterior domain with a nonlinear boundary condition on the interior boundary. Namely, we study positive radial solutions to:

$$
\left\{\begin{array}{l}
-\Delta u=\lambda k_{1}(|x|) f_{1}(u, v) \quad \text { on } \Omega, \\
-\Delta v=\lambda k_{2}(|x|) f_{2}(u, v) \quad \text { on } \Omega, \\
u(x)=v(x)=0 \quad \text { on }|x| \rightarrow \infty, \\
\frac{\partial u}{\partial \eta}+\tilde{c}_{1}(u) u=0 \quad \text { on }|x|=r_{0}, \\
\frac{\partial v}{\partial \eta}+\tilde{c}_{2}(v) v=0, \quad \text { on }|x|=r_{0} .
\end{array}\right.
$$

where $\Delta u=\operatorname{div}(\nabla u)$ and $\Delta v=\operatorname{div}(\nabla v)$ are the Laplacian of $u, \lambda$ is a positive parameter, $\Omega=\left\{x \in \mathbb{R}^{n}: N>2,|x|>r_{0}, r_{0}>0\right\}$, let $i=[1,2]$ then $K_{i}:\left[r_{0}, \infty\right) \rightarrow(0, \infty)$ is a continuous function such that $\lim _{r \rightarrow \infty} k_{i}(r)=0$ and $\frac{\partial}{\partial \eta}$ is the outward normal derivative, and $\tilde{c}_{i}:[0, \infty) \rightarrow(0, \infty)$ is a is an non decreasing (increasing) function. Here the reaction term $f_{i}:[0, \infty) \times[0, \infty) \rightarrow R$ is a $C^{1}$ function. The case when $f_{i}<0$ (see [1] [2], that the study of positive solutions to such problems is considerably more challenging than in the case $f_{i}>0$ (positone problems). For a rich history on semipositone problems with Dirichlet boundary conditions on bounded domains, (see [3]-[8], and on domains exterior to a ball, see [9] [10] [11]. Such nonlinear boundary conditions occur very naturally in applications see [12] for a detailed description in a model arising in combustion theory. Recently, the existence of a radial positive solution for (1.1) when $\lambda \gg 1$ has been established in [13], via the method of subsuper solutions. Here we discuss the uniqueness of this radial solution when some additional assumptions hold. In [14], the authors study such a uniqueness result for the case of Dirichlet boundary condition on $|x|=r_{0}$. Our focus in this paper is to consider the uniqueness result for semipositone problem when a class of nonlinear boundary condition is satisfied at $|x|=r_{0}$. The fact that we have no longer a fixed value of $u$ on $|x|=r_{0}$ results in quite a challenge in extending the results in [15].

Namely, we need to establish a detailed behavior of $u$ at $|x|=r_{0}$ to achieve our goal. Instead of working directly with (1), we note that the change of variables $r=|x|$ and $s=\left(\frac{r}{r_{0}}\right)^{2-N}$ transforms (1) into the following boundary value problem:

$$
\left\{\begin{array}{l}
-u^{\prime \prime}(t)=\lambda \tilde{a}_{1}(t) f_{1}(u(t), v(t)) \quad t \in[0,1], \\
-v^{\prime \prime}(t)=\lambda \tilde{a}_{2}(t) f_{2}(u(t), v(t)) \quad t \in[0,1], \\
\frac{N-2}{r_{0}} u^{\prime}+\tilde{c}_{1}(u(1)) u(1)=0, \\
\frac{N-2}{r_{0}} v^{\prime}+\tilde{c}_{2}(v(1)) v(1)=0, \\
u(0)=v(0)=0 .
\end{array}\right.
$$


where $\quad \tilde{a}_{i}=\frac{r_{0}^{2}}{(2-N)^{2}} t^{\frac{-2(N-1)}{N-2}} k_{i}\left(r_{0} t^{\frac{1}{2-N}}\right)$. We will only assume $k_{i} \leq \frac{1}{r^{N+\mu}}$ for $r \gg 1$ and for some $\mu \in(0, N-2)$. then $\tilde{a}_{i} \in((0,1],(0, \infty))$ could be singular at 0. if $\mu \geq N-2, \quad \tilde{a}_{i}$ will be nonsingular at 0 and it will be an easier case to study. Note that $\tilde{a}_{i}=\inf _{t \in(0,1]} \tilde{a}_{i}(t)>0$ and there exists a constant $\tilde{d}>0$ such that $\tilde{a}_{i} \leq \frac{\tilde{d}}{t^{\alpha}}$ for all $t \in(0,1]$ where $\tilde{\alpha}=\frac{(N-2)-\mu}{N-2}$. Motivated by the above discussion, in this paper, we will study positive solutions in $C^{2}(0,1) \cap C^{1}[0,1]$ to the following boundary value problems:

$$
\left\{\begin{array}{l}
-u^{\prime \prime}(t)=\lambda a_{1}(t) f_{1}(u(t), v(t)) \quad t \in[0,1] \\
-v^{\prime \prime}(t)=\lambda a_{2}(t) f_{2}(u(t), v(t)) \quad t \in[0,1] \\
u^{\prime}(1)+c_{1}(u(1)) u(1)=0 \\
v^{\prime}(1)+c_{2}(v(1)) v(1)=0 \\
u(0)=v(0)=0
\end{array}\right.
$$

where $c_{i}:[0, \infty) \rightarrow(0, \infty)$ is a continuous function and $a_{i} \in C((0,1],(0, \infty))$ is such that:

(H1) $a_{i}=\inf _{t \in(0,1]} a_{i}(t)>0$;

(H2) there exists a constant $d>0$ such that $a_{i}(t)=\frac{d}{t^{\alpha}}$ for all $t \in(0, \varepsilon]$ where $a \in(0,1)$ and $\varepsilon \approx 0$

(H3) $a_{i}$ is decreasing. We consider various $C^{1}$ classes of the reaction term $f_{i}:[0, \infty) \times[0, \infty) \rightarrow R$ satisfying the following:

(F1) $f_{i}<0$ and $\lim _{s \rightarrow \infty} \frac{f_{i}(s)}{s}=0 ; i=1,2$

(F2) $f_{i}$ is increasing and $\lim _{s \rightarrow \infty} f_{i}(s)=\infty ; i=1,2$

(F3) $f_{i}$ is concave on $[0, \infty), i=1,2$

Theorem 1.1. Assume (H1) - (H3) and (F1) - (F3). Then (1.3) has a unique positive solution for all $\lambda$ sufficiently large.

In Section two we will establish important a priori estimates. We will first recall some important results from [8] where the authors studied the case of Dirichlet boundary condition, or equivalently (1.3) with the boundary condition $t=1$ replaced by $u(1)=v(1)=0$. These results do not depend on the boundary condition at $t=1$ and hence it is also true for solutions of (1.3). In view of the readers convenience we include the proofs of these results. In Section three, we prove Theorem 1.1.

\section{Advance Estimates}

Let $F(s)=\int_{0}^{s} f_{i}(t)$ ds . Note that there exist unique positive numbers $\beta, \theta$ such that $f_{i}(\beta)=0$ and $F(\theta)=0$ and $\beta<\theta$

Theorem 2.1. (See [8].) Let $u, v$ are a positive solution of (1.3). Then $u$ and $v$ has only one interior maximum in $(0,1)$, say at $t_{m}$, depending on $\lambda$, and $u\left(t_{m}\right)>\theta, v\left(t_{m}\right)>\theta$.

Proof. Let 


$$
\left\{\begin{array}{l}
E_{1}(t)=\lambda F(u(t)) a_{1}(t)+\frac{\left|u^{\prime}(t)\right|^{2}}{2}, t \in(0,1), \\
E_{2}(t)=\lambda F(v(t)) a_{2}(t)+\frac{\left|v^{\prime}(t)\right|^{2}}{2}, t \in(0,1)
\end{array}\right.
$$

then

$$
\left\{\begin{array}{l}
E_{1}^{\prime}(t)=\lambda F(u(t)) a_{1}^{\prime}(t), t \in(0,1), \\
E_{2}^{\prime}(t)=\lambda F(v(t)) a_{2}^{\prime}(t), t \in(0,1)
\end{array}\right.
$$

Note that by $(\mathrm{H} 3), a_{1}^{\prime}(t)<0$ and $a_{2}^{\prime}(t)<0$ for all $t \in(0,1]$. Hence, $E_{1}(t)$ and $E_{2}(t)$ are increases when $u(t)<\theta, v(t)<\theta$ and decreases when $u(t)>\theta, \quad v(t)>\theta$.

Let $t_{m} \in(0,1)$ be the first point at which $\mathrm{u}$ has a local maximum and assume that $u(t) \leq \theta$ and $v(t) \leq \theta$ for all $t \in\left[0, t_{m}\right]$. Then $E_{1}(t)$ and $E_{2}(t)$ are increases in $\left[0, t_{m}\right]$. Now integrating (1.3) from $t$ to $t_{m}$, for $t<t_{m}$

$$
\left\{\begin{array}{l}
u^{\prime}(t)=\int_{t}^{t_{m}} \lambda a_{1}(s) f_{1}(u(s)) \mathrm{d} s \leq \lambda f_{1}(\theta) \int_{t}^{t_{m}} \frac{c_{1}}{s^{\alpha}} \mathrm{d} s \leq \lambda \frac{c_{1} f_{1}(\theta)}{1-\alpha} \\
v^{\prime}(t)=\int_{t}^{t_{m}} \lambda a_{2}(s) f_{2}(v(s)) \mathrm{d} s \leq \lambda f_{2}(\theta) \int_{t}^{t_{m}} \frac{c_{2}}{s^{\alpha}} \mathrm{d} s \leq \lambda \frac{c_{2} f_{2}(\theta)}{1-\alpha}
\end{array}\right.
$$

where $c_{i}>d$ are such that $a_{i}(t) \leq \frac{c_{i}}{t^{\alpha}}$ for all $t \in(0,1)$ using (H2). Integrating again (1.6) from 0 to $t, t \leq t_{m}$

$$
\begin{cases}u(t) \leq \int_{0}^{t} \lambda \frac{c_{1} f_{1}(\theta)}{1-\alpha} \mathrm{d} s, & C_{0}=\frac{c_{1} f_{1}(\theta)}{1-\alpha} \\ V(t) \leq \int_{0}^{t} \lambda \frac{C_{2} f_{2}(\theta)}{1-\alpha} \mathrm{d} s, & C_{0}=\frac{c_{2} f_{2}(\theta)}{1-\alpha}\end{cases}
$$

Since $f_{i}$ are continuous, there exists $K_{0}>0$ such that $|F(u)| \leq K_{0} u$ and $|F(v)| \leq K_{0} v$ for all $(u, v) \in[0, \theta]$. Hence

$$
\begin{aligned}
& \lim _{t \rightarrow 0^{+}} \lambda|F(u(t))| a_{1}(t) \leq \lim _{t \rightarrow 0^{+}} \lambda K_{0} u(t) a_{1}(t) \leq \lim _{t \rightarrow 0^{+}} \lambda^{2} K_{0} C_{0} c_{1} d t^{1-\alpha}=0 \\
& \lim _{t \rightarrow 0^{+}} \lambda|F(v(t))| a_{2}(t) \leq \lim _{t \rightarrow 0^{+}} \lambda K_{0} v(t) a_{2}(t) \leq \lim _{t \rightarrow 0^{+}} \lambda^{2} K_{0} C_{0} c_{2} d t^{1-\alpha}=0
\end{aligned}
$$

Hence $\lim _{t \rightarrow 0^{+}} E_{i}(t) \geq 0$. Since $E_{i}(t)$ increases on $\left[0, t_{m}\right]$, $E_{1}\left(t_{m}\right)=\lambda F\left(u\left(t_{m}\right)\right) a_{1}\left(t_{m}\right)>0$ and $E_{2}\left(t_{m}\right)=\lambda F\left(v\left(t_{m}\right)\right) a_{2}\left(t_{m}\right)>0$ and which is a contra-diction if $u\left(t_{m}\right) \leq \theta$ and $v\left(t_{m}\right) \leq \theta$. Suppose that $u$ and $v$ has two interior maxima. Then there exists $\tilde{t} \in(\tilde{t}, 1)$ such that $u^{\prime}(\tilde{t})=0, v^{\prime}(\tilde{t})=0$ and $u^{\prime \prime}(\tilde{t}) \geq 0, v^{\prime \prime}(\tilde{t}) \geq 0$. Since $u^{\prime \prime}(\tilde{t})=\lambda a_{1}(\tilde{t}) f_{1}(u(\tilde{t})) \geq 0$, and $v^{\prime \prime}(\tilde{t})=\lambda a_{2}(\tilde{t}) f_{2}(v(\tilde{t})) \geq 0$ we have $f_{1}(u(\tilde{t})) \leq 0$ and $f_{2}(v(\tilde{t})) \leq 0$ which implies that $u(\tilde{t}) \leq \beta$ and $u(\tilde{t}) \leq \beta$. Thus $E_{i}(\tilde{t})<0$. Let $t_{\theta}\left(t_{m}, \tilde{t}\right)$ such that $u\left(t_{\theta}\right)=\theta$ and $v\left(t_{\theta}\right)=\theta$. Then $E_{1}\left(t_{\theta}\right)=\frac{\left|u^{\prime}\left(t_{\theta}\right)\right|^{2}}{2} \geq 0, \quad E_{2}\left(t_{\theta}\right)=\frac{\left|v^{\prime}\left(t_{\theta}\right)\right|^{2}}{2} \geq 0$ and $E_{i}$ increases in $\left(t_{\theta}, \tilde{t}\right)$ since $u(t)<\theta$ and since $v(t)<\theta$ in $\left(t_{\theta}, \tilde{t}\right)$. Hence $E_{i}(\tilde{t})>0$, which is a contradiction. Therefore, we have only one interior maximum and that maximum value is larger than $\theta$. 
Theorem 2.2. (See [8].) Let $u$ and $v$ be a positive solution of (1.3) and let $t_{\beta} \in\left(0, t_{m}\right)$ such that $u\left(t_{\beta}\right)=\beta$ and $v\left(t_{\beta}\right)=\beta$. Then $t_{\beta} \leq O\left(\lambda^{-\frac{1}{2}}\right)$ as $\lambda \rightarrow \infty$.

Proof. Let $t_{\frac{\beta}{2}} \in\left(0, t_{\beta}\right)$ be the point such that $u\left(t_{\frac{\beta}{2}}\right)=\frac{\beta}{2}$ and $v\left(t_{\frac{\beta}{2}}\right)=\frac{\beta}{2}$ from Integrating (1.3) from 0 to $t$ for some $t<t_{\frac{\beta}{2}}$

$$
\begin{aligned}
& u^{\prime}(t)=u^{\prime}(0)-\lambda \int_{0}^{t} a_{1}(s) f_{1}(u(s)) \mathrm{d} s \geq \lambda \underline{a_{1}}\left(-f_{1}\left(\frac{\beta}{2}\right)\right) t \\
& v^{\prime}(t)=v^{\prime}(0)-\lambda \int_{0}^{t} a_{2}(s) f_{2}(v(s)) \mathrm{d} s \geq \lambda \underline{a_{2}}\left(-f_{2}\left(\frac{\beta}{2}\right)\right) t,
\end{aligned}
$$

Integrating the above again from 0 to $t_{\beta}$

$$
t_{\frac{\beta}{2}} \leq \tilde{c}_{1} \lambda^{-\frac{1}{2}}, t_{\frac{\beta}{2}} \leq \tilde{c}_{2} \lambda^{-\frac{1}{2}} \text { where }\left\{\begin{array}{l}
\tilde{c}_{1}=\left(\frac{-\beta}{a_{1}\left(-f_{1}\left(\frac{\beta}{2}\right)\right)^{\frac{1}{2}}}\right)>0 \\
\tilde{c}_{2}=\left(\frac{-\beta}{a_{2}\left(-f_{2}\left(\frac{\beta}{2}\right)\right)^{\frac{1}{2}}}\right)>0
\end{array}\right.
$$

By the mean value theorem, there exists a $\tilde{t} \in\left[0, t_{\frac{\beta}{2}}\right]$ such that

$u\left(t_{\frac{\beta}{2}}\right)-u(0)=u^{\prime}(\tilde{t}) t_{\frac{\beta}{2}}, \quad v\left(t_{\frac{\beta}{2}}\right)-v(0)=v^{\prime}(\tilde{t}) t_{\frac{\beta}{2}}$ and by (2.5) $u^{\prime}(\tilde{t}) \geq \frac{\beta}{2 \tilde{c}_{1}} \lambda^{\frac{1}{2}}$ and $v^{\prime}(\tilde{t}) \geq \frac{\beta}{2 \tilde{c}_{2}} \lambda^{\frac{1}{2}}$. Since $u^{\prime}$ and $v^{\prime}$ are increases in $\left[0, t_{\beta}\right]$,

$$
\begin{cases}u^{\prime}(t) \geq \frac{\beta}{2 \tilde{c}_{1}} \lambda^{\frac{1}{2}}, & t \in\left[t_{\frac{\beta}{2}}, t_{\beta}\right] \\ v^{\prime}(t) \geq \frac{\beta}{2 \tilde{c}_{2}} \lambda^{\frac{1}{2}}, & t \in\left[t_{\frac{\beta}{2}}, t_{\beta}\right]\end{cases}
$$

Integrating (2.6) from $t_{\frac{\beta}{2}}$ to $t_{\beta}$, we have that $\left(t_{\beta}-t_{\frac{\beta}{2}}\right) \leq \tilde{c}_{1} \lambda^{-\frac{1}{2}}$ and $\left(t_{\beta}-t_{\frac{\beta}{2}}\right) \leq \tilde{c}_{1} \lambda^{-\frac{1}{2}}$. This implies $\left.t_{\beta} \leq \mathcal{O} \lambda^{-\frac{1}{2}}\right)$ by (2.5).

Lemma 2.3. Let $u$ and $v$ be a positive solution of (1.3). Then $u(1) \rightarrow \infty$ and $v(1) \rightarrow \infty$ as $\lambda \rightarrow \infty$

Proof. We first claim that $u(1) \geq \frac{\beta+\theta}{2}$ and $v(1) \geq \frac{\beta+\theta}{2}$ for $\lambda \gg 1$. As- 
sume that $u(1)<\frac{\beta+\theta}{2}$ and $v(1)<\frac{\beta+\theta}{2}$ Then there exists a $\tilde{t}_{\theta} \in\left(t_{m}, 1\right)$ such that $u\left(\tilde{t}_{\theta}\right)=\theta$ and $v\left(\tilde{t}_{\theta}\right)=\theta$ where $t_{m}$ is the point at which $u, v$ achieves are maximum, and $u\left(t_{m}\right)>\theta, v\left(t_{m}\right)>\theta$ by Lemma 2.1.

From (2.1) and (2.2), $E_{1}\left(\tilde{t}_{\theta}\right)=\frac{\left|u^{\prime}\left(\tilde{t}_{\theta}\right)\right|}{2}>0, \quad E_{2}\left(\tilde{t}_{\theta}\right)=\frac{\left|v^{\prime}\left(\tilde{t}_{\theta}\right)\right|}{2}>0$ and $E_{i}(t) \geq 0$ on $\left[\tilde{t}_{\theta}, 1\right]$ since $u(t) \leq \theta$ and $v(t) \leq \theta$ in $\left[\tilde{t}_{\theta}, 1\right]$. Hence we obtain that

$$
\begin{aligned}
& E_{1}(1)=\lambda F(u(1)) a_{1}(1)+\frac{\left|u^{\prime}(1)\right|}{2}>0, \\
& E_{2}(1)=\lambda F(v(1)) a_{2}(1)+\frac{\left|v^{\prime}(1)\right|}{2}>0
\end{aligned}
$$

and from (1.3), we have

$$
\left\{\begin{array}{l}
c_{1}(u(1)) u(1)=-u^{\prime}(1) \geq \sqrt{-2 \lambda F(u(1)) a_{1}(1)}, \\
c_{2}(v(1)) v(1)=-v^{\prime}(1) \geq \sqrt{-2 \lambda F(v(1)) a_{2}(1)} .
\end{array}\right.
$$

This cannot hold unless $u(1) \rightarrow 0$ and $v(1) \rightarrow 0$ as $\lambda \rightarrow \infty$. However, rewriting (1.10) as

$$
\left\{\begin{array}{l}
c_{1}(u(1)) u(1)^{\frac{1}{2}} \geq \sqrt{-2 \lambda \frac{F(u(1))}{u(1)} a_{1}(1),} \\
c_{2}(v(1)) v(1)^{\frac{1}{2}} \geq \sqrt{-2 \lambda \frac{F(v(1))}{v(1)} a_{2}(1) .}
\end{array}\right.
$$

we obtain a contradiction when $\lambda \gg 1$ since $\frac{F(u(1))}{u(1)} \rightarrow f_{1}(0)$,

$\frac{F(v(1))}{v(1)} \rightarrow f_{2}(0)$ if $u(1) \rightarrow 0$ and $v(1) \rightarrow 0$ as $\lambda \rightarrow \infty$. Hence,

$$
u(1) \geq \frac{\beta+\theta}{2} \text { and } v(1) \geq \frac{\beta+\theta}{2} \text { for } \lambda \gg 1
$$

Next, we claim that $u\left(t_{m}\right)=\|u\|_{\infty} \rightarrow \infty$ and $v\left(t_{m}\right)=\|v\|_{\infty} \rightarrow \infty$ as $\lambda \rightarrow \infty$. Let

$h:=u-\beta$ and $w:=v-\beta$ then $h>0, w>0$ in $\left(t_{\beta}, 1\right]$ and satisfies

\[ \left\{\begin{array}{l}-h^{\prime \prime}=\lambda a_{1}(t) \frac{f_{1}(u)}{u-\beta} h \quad\left(t_{\beta}, 1\right) \\ -w^{\prime \prime}=\lambda a_{2}(t) \frac{f_{2}(v)}{v-\beta} w \quad\left(t_{\beta}, 1\right) \\ h\left(t_{\beta}\right)=w\left(t_{\beta}\right)=0, \\ h(1)=u(1)-\beta>0 \\ w(1)=v(1)-\beta>0\end{array}\right. \]
Let $\psi=\sin \left(\frac{\pi\left(t-t_{\beta}\right)}{t-t_{\beta}}\right)$. Then $\psi$ satisfies: 


$$
\left\{\begin{array}{l}
-\psi^{\prime \prime}=\frac{\pi^{2}}{\left(t-t_{\beta}\right)^{2}} \psi, \quad\left(t_{\beta}, 1\right) \\
\psi\left(t_{\beta}\right)=\psi(1)=0
\end{array}\right.
$$

Multiplying (1.13) by $\psi$ and (1.14) by $h$,and $w$ integrating both from $t_{\beta}$ to 1 and subtracting, we have

$$
\begin{aligned}
& \int_{t_{\beta}}^{1}\left(h^{\prime \prime} \psi-\psi^{\prime \prime} h\right) \mathrm{d} t=\int_{t_{\beta}}^{1}\left(\frac{\pi^{2}}{\left(1-t_{\beta}\right)^{2}}-\lambda \frac{f_{1}(u)}{u-\beta} a_{1}(t)\right) h \psi \mathrm{d} t, \\
& \int_{t_{\beta}}^{1}\left(w^{\prime \prime} \psi-\psi^{\prime \prime} w\right) \mathrm{d} t=\int_{t_{\beta}}^{1}\left(\frac{\pi^{2}}{\left(1-t_{\beta}\right)^{2}}-\lambda \frac{f_{2}(v)}{v-\beta} a_{2}(t)\right) w \psi \mathrm{d} t
\end{aligned}
$$

Since $\int_{t_{\beta}}^{1}\left(h^{\prime \prime} \psi-\psi^{\prime \prime} h\right) \mathrm{d} t=-\psi^{\prime}(1) h(1)(>0)$ and $\int_{t_{\beta}}^{1}\left(w^{\prime \prime} \psi-\psi^{\prime \prime} w\right) \mathrm{d} t=-\psi^{\prime}(1) w(1)(>0)$ by integration by parts, we can see that $\frac{\pi^{2}}{\left(1-t_{\beta}\right)^{2}}>\lambda \frac{f_{1}(u)}{u-\beta} a_{1}(t)$ and $\frac{\pi^{2}}{\left(1-t_{\beta}\right)^{2}}>\lambda \frac{f_{2}(v)}{v-\beta} a_{2}(t)$ for some $t \in\left(t_{\beta}, 1\right)$

Note that $\inf _{(0,1]} a_{1}(t)>0, \inf _{(0,1]} a_{2}(t)>0$ and from Lemma 2.2 we can assume $\left(1-t_{\beta}\right)>\frac{1}{2}$. Thus (2.11) is only true if $\frac{f_{1}(u)}{u-\beta} \rightarrow 0$ and $\frac{f_{2}(v)}{v-\beta} \rightarrow 0$ when $\lambda \gg 1$. By (F1) (F2), we conclude that $u\left(t_{m}\right)=\|u\|_{\infty}$ and $v\left(t_{m}\right)=\|v\|_{\infty}$ as $\lambda \rightarrow \infty$. Notice that since $u^{\prime \prime}<0$ and $v^{\prime \prime}<0$ in $\left(t_{\beta}, 1\right]$, we have

$$
\begin{gathered}
u(t) \geq \frac{u\left(t_{m}\right)-\beta}{t_{m}-t_{\beta}}\left(t-t_{\beta}\right)+\beta, \quad t \in\left[t_{\beta}, t_{m}\right] \\
v(t) \geq \frac{v\left(t_{m}\right)-\beta}{t_{m}-t_{\beta}}\left(t-t_{\beta}\right)+\beta, \quad t \in\left[t_{\beta}, t_{m}\right] \\
u(t) \geq \frac{u\left(t_{m}\right)-\frac{\beta+\theta}{2}}{1-t_{m}}(1-t)+\frac{\beta+\theta}{2}, \quad t \in\left[t_{m}, 1\right] \\
v(t) \geq \frac{v\left(t_{m}\right)-\frac{\beta+\theta}{2}}{1-t_{m}}(1-t)+\frac{\beta+\theta}{2}, \quad t \in\left[t_{m}, 1\right]
\end{gathered}
$$

Since $u\left(t_{m}\right) \rightarrow \infty, v\left(t_{m}\right) \rightarrow \infty$ and $t_{\beta} \rightarrow 0$ as $\lambda \rightarrow \infty$, it is all true that

$$
v(t) \geq \frac{\beta+\theta}{2} \text { and } u(t) \geq \frac{\beta+\theta}{2} \text {, in }\left[\frac{1}{4}, 1\right] \text { for } \lambda \gg 1
$$

Now we show that $u(1) \rightarrow \infty$ and $v(1) \rightarrow \infty$ as $\lambda \rightarrow \infty$. Since $u, v$ is a solution of (1.3), $u$ and $v$ can be written as: (see Appendix 8.2 in [5] for details)

$$
\begin{aligned}
& u(t)=\lambda \int_{0}^{1} G(t, s) a_{1}(s) f_{1}(u(s)) \mathrm{d} s-c_{1}(u(1)) u(1) t \\
& v(t)=\lambda \int_{0}^{1} G(t, s) a_{2}(s) f_{2}(v(s)) \mathrm{d} s-c_{2}(v(1)) v(1) t
\end{aligned}
$$

where 


$$
G(t, s)= \begin{cases}s, & 0 \leq s \leq t \leq 1 \\ t, & 0 \leq t \leq s \leq 1\end{cases}
$$

Let $t=1$. Then from (1.17) and (1.18), we have

$$
\begin{aligned}
& {\left[1+c_{1}(u(1))\right] u(1)} \\
& =\lambda\left[\int_{0}^{t_{\beta}} G(1, s) a_{1}(s) f_{1}(u(s)) \mathrm{d} s+\int_{t_{\beta}}^{1} G(1, s) a_{1}(s) f_{1}(u(s)) \mathrm{d} s\right] \\
& {\left[1+c_{2}(v(1))\right] v(1)} \\
& =\lambda\left[\int_{0}^{t_{\beta}} G(1, s) a_{2}(s) f_{2}(v(s)) \mathrm{d} s+\int_{t_{\beta}}^{1} G(1, s) a_{2}(s) f_{2}(v(s)) \mathrm{d} s\right]
\end{aligned}
$$

Then using the fact $G(1, s)=s$ and $t_{\beta} \rightarrow \infty$ as $\lambda \rightarrow \infty$, for $\lambda$ large we obtain

$$
\begin{aligned}
{\left[1+c_{1}(u(1))\right] u(1) } & =\lambda\left(\int_{0}^{t_{\beta}} s a_{1}(s) f_{1}(u(s)) \mathrm{d} s+\int_{t_{\beta}}^{1} s a_{1}(s) f_{1}(u(s)) \mathrm{d} s\right) \\
{\left[1+c_{2}(v(1))\right] v(1) } & =\lambda\left(\int_{0}^{t_{\beta}} s a_{2}(s) f_{2}(v(s)) \mathrm{d} s+\int_{t_{\beta}}^{1} s a_{2}(s) f_{2}(v(s)) \mathrm{d} s\right) \\
& \geq \lambda\left(\int_{0}^{t_{\beta}} s a_{1}(s) f_{1}(u(s)) \mathrm{d} s+\int_{\frac{1}{4}}^{1} s a_{1}(s) f_{1}(u(s)) \mathrm{d} s\right) \\
& \geq \lambda\left(\int_{0}^{t_{\beta}} s a_{2}(s) f_{2}(v(s)) \mathrm{d} s+\int_{\frac{1}{4}}^{1} s a_{2}(s) f_{2}(v(s)) \mathrm{d} s\right) \\
& \geq \frac{\lambda}{2} f_{1}\left(\frac{\beta+\theta}{2}\right) \int_{\frac{1}{4}}^{1} s a_{1}(s) \mathrm{d} s \\
& \geq \frac{\lambda}{2} f_{2}\left(\frac{\beta+\theta}{2}\right) \int_{\frac{1}{4}}^{1} s a_{2}(s) \mathrm{d} s
\end{aligned}
$$

where the last inequality is obtained by (1.16). Hence, we have

$$
\left[1+c_{1}(u(1))\right] u(1) \geq \lambda K \text { and }\left[1+c_{2}(v(1))\right] v(1) \geq \lambda K,
$$

where $K=\frac{1}{2} f_{1}\left(\frac{\beta+\theta}{2}\right) \int_{\frac{1}{4}}^{1} s a_{1}(s) \mathrm{d} s>0$ and $K=\frac{1}{2} f_{2}\left(\frac{\beta+\theta}{2}\right) \int_{\frac{1}{4}}^{1} s a_{2}(s) \mathrm{d} s>0$.

Now, from (1.21), clearly $u(1) \rightarrow \infty, v(1) \rightarrow \infty$ as $\lambda \rightarrow \infty$.

Lemma 2.4. Let $u$ and $v$ be a positive solution of (1.3). Then there exists $[\alpha, \mu] \subset\left[\frac{1}{4}, 1\right], \alpha \neq \mu$, both independent of $\lambda$, such that $\inf _{[\alpha, \mu]} u(t) \rightarrow \infty$ and $\inf _{[\alpha, \mu]} v(t) \rightarrow \infty$ as $\lambda \rightarrow \infty$

Proof. As $\lambda \rightarrow \infty, t_{m}$ may converge to 1 or to any other point in $(0,1)$. First we consider the case $t_{m} \nrightarrow 1$ as $\lambda \rightarrow \infty$. Since $u(1)<u\left(t_{m}\right)$ and $v(1)<v\left(t_{m}\right)$ clearly there exists $\alpha<1$ such that $\inf _{[\alpha, 1]} u(t) \rightarrow \infty$ and $\inf _{[\alpha, 1]} v(t) \rightarrow \infty$ as $\lambda \rightarrow \infty$ by Lemma 2.3.

Now, let us consider the case when $t_{m} \rightarrow 1$ as $\lambda \rightarrow \infty$. By differentiating (1.17) and (1.18) (or integrating (1.3)), we obtain

$$
\begin{gathered}
u^{\prime}(t)=\lambda \int_{t}^{1} a_{1}(s) f_{1}(u(s)) \mathrm{d} s-c_{1}(u(1)) u(1), \quad t \in[0,1], \\
v^{\prime}(t)=\lambda \int_{t}^{1} a_{2}(s) f_{2}(v(s)) \mathrm{d} s-c_{2}(v(1)) v(1), \quad t \in[0,1]
\end{gathered}
$$

which gives us that 


$$
\begin{aligned}
& u^{\prime}(t)=\lambda \int_{t}^{1} a_{1}(s) f_{1}(u(s)) \mathrm{d} s-c_{1}(u(1)) u(1)=0, \\
& v^{\prime}(t)=\lambda \int_{t_{m}}^{1} a_{2}(s) f_{2}(v(s)) \mathrm{d} s-c_{2}(v(1)) v(1)=0
\end{aligned}
$$

Now we rewrite (1.17) and (1.18) by using (1.22), (1.23) as:

$$
\begin{aligned}
u(t)= & \lambda \int_{0}^{1} G(t, s) a_{1}(s) f_{1}(u(s)) \mathrm{d} s-\lambda\left(\int_{t_{m}}^{1} a_{1}(s) f_{1}(u(s)) \mathrm{d} s\right) t, \\
v(t)= & \lambda \int_{0}^{1} G(t, s) a_{2}(s) f_{2}(v(s)) \mathrm{d} s-\lambda\left(\int_{t_{m}}^{1} a_{2}(s) f_{2}(v(s)) \mathrm{d} s\right) t \\
= & \lambda \int_{0}^{t_{\beta}} G(s, t) a_{1}(s) f_{1}(u(s)) \mathrm{d} s+\lambda \int_{t_{\beta}}^{t_{m}} G(t, s) a_{1}(s) f_{1}(u(s)) \mathrm{d} s \\
& +\lambda \int_{t_{m}}^{1}[G(t, s)-t] a_{1}(s) f_{1}(u(s)) \mathrm{d} s . \\
= & \lambda \int_{0}^{t_{\beta}} G(s, t) a_{2}(s) f_{2}(v(s)) \mathrm{d} s+\lambda \int_{t_{\beta}}^{t_{m}} G(t, s) a_{2}(s) f_{2}(v(s)) \mathrm{d} s \\
& +\lambda \int_{t_{m}}^{1}[G(t, s)-t] a_{2}(s) f_{2}(v(s)) \mathrm{d} s .
\end{aligned}
$$

Note that if $t \in\left[0, t_{m}\right]$, then

$$
\int_{t_{m}}^{1}[G(t, s)-t] a_{1}(s) f_{1}(u(s)) \mathrm{d} s=0
$$

and

$$
\int_{t_{m}}^{1}[G(t, s)-t] a_{2}(s) f_{2}(v(s)) \mathrm{d} s=0
$$

since $t \leq t_{m} \leq s \leq 1$ implies $G(t, s)=t$. Now $t_{\beta} \rightarrow 0$ and $t_{m} \rightarrow 1$ as $\lambda \rightarrow \infty$. Hence, for $t \in\left[\frac{1}{4}, \frac{3}{4}\right]$ and $\lambda$ large we obtain

$$
\begin{aligned}
u(t) & =\lambda\left(\int_{0}^{t_{\beta}} G(t, s) a_{1}(s) f_{1}(u(s)) \mathrm{d} s+\int_{t_{\beta}}^{t_{m}} G(t, s) a_{1}(s) f_{1}(u(s)) \mathrm{d} s\right), \\
v(t) & =\lambda\left(\int_{0}^{t_{\beta}} G(t, s) a_{2}(s) f_{2}(v(s)) \mathrm{d} s+\int_{t_{\beta}}^{t_{m}} G(t, s) a_{2}(s) f_{2}(v(s)) \mathrm{d} s\right) \\
& \geq \lambda\left(\int_{0}^{t_{\beta}} G(t, s) a_{1}(s) f_{1}(u(s)) \mathrm{d} s+\int_{\frac{1}{4}}^{\frac{3}{4}} G(t, s) a_{1}(s) f_{1}(u(s)) \mathrm{d} s\right), \\
& \geq \lambda\left(\int_{0}^{t_{\beta}} G(t, s) a_{2}(s) f_{2}(v(s)) \mathrm{d} s+\int_{\frac{1}{4}}^{\frac{3}{4}} G(t, s) a_{2}(s) f_{2}(v(s)) \mathrm{d} s\right) \\
& \geq \lambda \frac{a_{1}}{2} f_{1}\left(\frac{\beta+\theta}{2}\right) \int_{\frac{1}{4}}^{\frac{3}{4}} G(t, s) \mathrm{d} s, \\
& \geq \lambda \frac{a_{2}}{2} f_{2}\left(\frac{\beta+\theta}{2}\right) \int_{\frac{1}{4}}^{\frac{3}{4}} G(t, s) \mathrm{d} s .
\end{aligned}
$$

Thus,

$$
u(t) \geq \lambda \frac{a_{1}}{2} f_{1}\left(\frac{\beta+\theta}{2}\right) \inf _{\left[\frac{1}{4}, \frac{3}{4}\right]} \int_{\frac{1}{4}}^{\frac{3}{4}} G(t, s) \mathrm{d} s
$$

and

$$
v(t) \geq \lambda \frac{a_{2}}{2} f_{2}\left(\frac{\beta+\theta}{2}\right) \inf _{\left[\frac{1}{4}, \frac{3}{4}\right]} \int_{\frac{1}{4}}^{\frac{3}{4}} G(t, s) \mathrm{d} s
$$


$\underset{\lambda \rightarrow \infty}{\text { on }}\left[\frac{1}{4}, \frac{3}{4}\right]$, which means that $u(t) \rightarrow \infty$ and $v(t) \rightarrow \infty$ for all $t \in\left[\frac{1}{4}, \frac{3}{4}\right]$ as

Lemma 2.5. Let $u$ and $v$ be a positive solution of (1.3). Then there exists $\tilde{\lambda}$ such that if $\lambda>\tilde{\lambda}$, then

$$
u(t) \geq \lambda C d(t, \partial \Omega) \text { and } v(t) \geq \lambda C d(t, \partial \Omega)
$$

for some positive constant $C$, independent of $\lambda$. Here $\Omega=(0,1)$.

Proof. Let $\phi_{i}$ be the unique solution of the problems

$$
\left\{\begin{array}{l}
-\phi_{i}^{\prime \prime}=\omega_{\alpha, \mu} a_{i}(t), \quad\left(t_{\beta}, 1\right) \\
\psi_{i}\left(t_{\beta}\right)=\psi_{i}(1)=0, \text { and } i=1,2
\end{array}\right.
$$

where $\omega$ is the characteristic function. By the Hopf maximum principle there exists $\tilde{c}_{i}>0$ such that $\phi_{i}(t)>\tilde{c}_{i} e_{i}(t)$ for all $t \in[0,1]$, where $e_{i}$ are a solution of

$$
\left\{\begin{array}{l}
-e_{i}^{\prime \prime}=\omega_{\alpha, \mu} a_{i}(t), \quad\left(t_{\beta}, 1\right) \\
e_{i}(0)=e_{i}(1)=0, \text { and } i=1,2
\end{array}\right.
$$

Let $H>0$ be such that $D:=\tilde{c}_{i} f_{i}(H)+f_{i}(0)>0$, and this is possible by (F2). Let $u_{1}, v_{1}$ and $u_{2}, v_{2}$ satisfy

$$
\begin{aligned}
& -u_{1}^{\prime \prime}=\lambda f_{1}(H) \omega[\alpha, \mu] a_{1}(t), t \in(0,1), u_{1}(0)=0=u_{1}(1) \\
& -v_{1}^{\prime \prime}=\lambda f_{2}(H) \omega[\alpha, \mu] a_{2}(t), t \in(0,1), v_{1}(0)=0=v_{1}(1)
\end{aligned}
$$

and

$$
\begin{aligned}
& -u_{2}^{\prime \prime}=\lambda f_{1}(0) \omega[\alpha, \mu] a_{1}(t), t \in(0,1), u_{2}(0)=0=u_{2}(1) \\
& -v_{2}^{\prime \prime}=\lambda f_{2}(0) \omega[\alpha, \mu] a_{2}(t), t \in(0,1), v_{2}(0)=0=v_{2}(1)
\end{aligned}
$$

Now by Lemma 2.4 , there exists $\tilde{\lambda}>0$ such that if $\lambda>\tilde{\lambda}$, then

$$
u(t) \geq H \text { and } v(t) \geq H \text { on }[\alpha, \mu] .
$$

Hence, by (1.27), for $\lambda \gg 1$ we have that for $t \in(0,1)$

$$
\begin{aligned}
-u^{\prime \prime}=\lambda f_{1}(u) a_{1}(t) & \geq \lambda f_{1}(u) a_{1}(t) \omega\left[0, t_{B}\right]+\lambda f_{1}(u) a_{1}(t) \omega[\alpha, \mu], \\
-v^{\prime \prime}=\lambda f_{2}(v) a_{2}(t) & \geq \lambda f_{2}(v) a_{2}(t) \omega\left[0, t_{B}\right]+\lambda f_{2}(v) a_{2}(t) \omega[\alpha, \mu] \\
-v^{\prime \prime} & \geq \lambda f_{2}(0) a_{2}(t)+\lambda f_{2}(H) a_{2}(t) \omega[\alpha, \mu] \\
-u^{\prime \prime} & \geq \lambda f_{1}(0) a_{1}(t)+\lambda f_{1}(H) a_{1}(t) \omega[\alpha, \mu] \\
= & -\left(u_{1}-u_{2}\right)^{\prime \prime}(t) \\
= & -\left(v_{1}-v_{2}\right)^{\prime \prime}(t)
\end{aligned}
$$

$$
u(0)-\left(u_{1}-u_{2}\right)(0)=0, v(0)-\left(v_{1}-v_{2}\right)(0)=0 \text { and }
$$

$u(1)-\left(u_{1}-u_{2}\right)(1)=u(1)>0, v(1)-\left(v_{1}-v_{2}\right)(1)=v(1)>0$. By the maximum principle, $u(t)=u_{1}(t)-u_{2}(t)=\lambda f_{1}(H) \phi_{1}(t)+\lambda f_{1}(0) e_{1}(t)$ and

$$
\begin{array}{r}
v(t)=v_{1}(t)-v_{2}(t)=\lambda f_{2}(H) \phi_{2}(t)+\lambda f_{2}(0) e_{2}(t) \text { in }[0,1] . \text { Hence } \\
u(t) \geq f_{1}(H) \tilde{c}_{1} e_{1}(t)+\lambda f_{1}(0) e_{1}(t)=\lambda D e_{1}(t)
\end{array}
$$

and 


$$
v(t) \geq f_{2}(H) \tilde{c}_{2} e_{2}(t)+\lambda f_{2}(0) e_{2}(t)=\lambda D e_{2}(t)
$$

for all $t \in[0,1]$.

Note that there exists $L>0$ such that $e_{1}(t) \geq L d(t, \partial \Omega)$ and $e_{2}(t) \geq L d(t, \partial \Omega)$ for all $t \in[0,1]$. Hence, for $\lambda$ large $u(t) \geq \lambda C d(t, \partial \Omega)$ and $v(t) \geq \lambda C d(t, \partial \Omega)$ for all $t \in[0,1]$, where $C:=D L>0$.

Lemma 2.6. Let $u$ and $v$ be a positive solution of (1.3). Then there exists $H_{\lambda}$ such that $\|u\|_{\infty} \leq H_{\lambda}$ and $\|v\|_{\infty} \leq H_{\lambda}$.

Proof.

Let $B=\int_{0}^{1} a_{i}(s) \mathrm{d} s$. Then $B<\infty$ since $a_{i}(t) \leq c_{i} t_{\alpha}$ for all $t \in(0,1)$ for some $c_{i}>0$. Now for each given $\lambda>0$, there exists $W_{\lambda}>0$ such that if $W>W_{\lambda}$, then $\frac{f_{i}(W)}{W} \leq \frac{1}{2 \lambda B}$ due to the hypothesis (F1). Also since $f_{i} \in C^{1}([0, \infty), R)$, there exists $K_{\lambda}>0$ such that $f_{i}(W) \leq K_{\lambda}$ on $\left[0, W_{\lambda}\right]$. Hence,

$$
f_{i}(W) \leq \frac{W}{2 \lambda B}+K_{\lambda}, \quad W \in[0, \infty) .
$$

Now by Lemma 2.1 and (1.28), we have

$$
\begin{aligned}
\|u\|_{\infty} & =u\left(t_{m}\right)=\lambda \int_{0}^{1} G\left(t_{m}, s\right) a_{1}(s) f_{1}(u(s)) \mathrm{d} s-c_{1}(u(1)) u(1) t_{m} \\
\|v\|_{\infty} & =v\left(t_{m}\right)=\lambda \int_{0}^{1} G\left(t_{m}, s\right) a_{2}(s) f_{2}(v(s)) \mathrm{d} s-c_{2}(u(1)) u(1) t_{m} \\
& \leq \lambda \int_{0}^{1} G\left(t_{m}, s\right) a_{1}(s) f_{1}(u(s)) \mathrm{d} s \\
& \leq \lambda \int_{0}^{1} G\left(t_{m}, s\right) a_{2}(s) f_{2}(v(s)) \mathrm{d} s \\
& \leq \lambda \int_{0}^{1} G\left(t_{m}, s\right) a_{1}(s)\left[\frac{u\left(t_{m}\right)}{2 \lambda B}+K_{\lambda}\right] \mathrm{d} s \\
& \leq \lambda \int_{0}^{1} G\left(t_{m}, s\right) a_{2}(s)\left[\frac{v\left(t_{m}\right)}{2 \lambda B}+K_{\lambda}\right] \mathrm{d} s \\
& \leq \lambda \int_{0}^{1} a_{1}(s)\left[\frac{1}{2 \lambda B} u\left(t_{m}\right)+K_{\lambda}\right] \mathrm{d} s \\
& \leq \lambda \int_{0}^{1} a_{2}(s)\left[\frac{1}{2 \lambda B} v\left(t_{m}\right)+K_{\lambda}\right] \mathrm{d} s \quad(\text { since } G(t, s) \leq 1 \text { in }[0,1] \times[0,1]) \\
& =\frac{1}{2} u\left(t_{m}\right)+\lambda B K_{\lambda} \\
& =\frac{1}{2} v\left(t_{m}\right)+\lambda B K_{\lambda}
\end{aligned}
$$

Hence, for each $\lambda>0,\|u\|_{\infty} \leq H_{\lambda}$ and $\|v\|_{\infty} \leq H_{\lambda}$, where $H_{\lambda}=2 \lambda B K_{\lambda}$.

\section{Proof of Theorem 1.1}

We first claim that (1.3) has a maximal positive solutions $\tilde{u}, \tilde{v}$ for $\lambda \gg 1$. Let $\varphi_{i}$ be the solutions of the problems

$$
\left\{\begin{array}{l}
-\varphi_{1}^{\prime \prime}=a_{i}(t), \quad t \in(0,1) \\
\varphi_{i}^{\prime}(1)=c_{i}\left(\varphi_{i}(1)\right) \varphi_{i}(1)=0, \\
\varphi_{i}(0)=0 \quad \text { and } \quad i=1,2
\end{array}\right.
$$


Note that (1.29) has the unique solution since $e_{i}, \varphi_{0}$ are sub solutions and super solutions of (1.29), respectively, where $e_{i}$ is defined in (1.26) and $\varphi_{0}$ is the solutions of the linear boundary condition problems

$$
\left\{\begin{array}{l}
-\varphi_{0}^{\prime \prime}=a_{i}(t), \quad t \in(0,1) \\
\varphi_{0}^{\prime}(1)=c_{i}\left(\varphi_{0}(1)\right) \varphi_{0}(1)=0, \\
\varphi_{0}(0)=0 \text { and } i=1,2
\end{array}\right.
$$

Since $f_{i}$ satisfies (F1), given $\lambda>0$, we can choose $Z_{\lambda} \geq 1$ such that $Z_{\lambda}>\lambda f_{i}\left(Z_{\lambda}\left\|\varphi_{i}\right\|_{\infty}\right)$ and $Z_{\lambda}>\lambda f_{i}\left(H_{\lambda}\right)$ where $H_{\lambda}$ is as in Lemma 2.6. Then, $Z_{\lambda} \varphi_{i}$ are a super solutions of (1.3) since

$$
\left\{\begin{array}{l}
-\left(Z_{\lambda} \varphi_{i}\right)^{\prime \prime}=Z_{\lambda} a_{i}(t) \geq \lambda a_{i} f_{i}\left(Z_{\lambda}\left\|\varphi_{i}\right\|_{\lambda}\right) \geq \lambda a_{i} f_{i}\left(Z_{\lambda} \varphi_{i}\right), \quad t \in(0,1) \\
\left(Z_{\lambda} \varphi_{i}^{\prime}(1)\right)+c_{i}\left(Z_{\lambda}\left(\varphi_{i}(1)\right)\right) Z_{\lambda} \varphi_{i}(1)=Z_{\lambda}\left(\varphi_{i}^{\prime}(1)+c_{i}\left(Z_{\lambda}\left(\varphi_{i}(1)\right)\right) \varphi_{i}(1)\right) \\
\geq Z_{\lambda}\left(\varphi_{i}^{\prime}(1)+c_{i}\left(\varphi_{i}(1)\right) \varphi_{i}(1)\right)=0, \\
Z_{\lambda} \varphi_{i}(0)=0 \text { and } i=1,2
\end{array}\right.
$$

Next, we show that this super solution $Z_{\lambda} \varphi_{i}$ is larger than any positive solutions of (1.3). Let $\theta_{i}$ be any positive solutions of (1.3). From Lemma 2.6, we have

$$
\begin{aligned}
-\left(Z_{\lambda} \varphi_{i}-\theta_{i}\right)^{\prime \prime} & =Z_{\lambda} a_{i}(t)-\lambda a_{i} f_{i}\left(\theta_{i}\right)=a_{i}\left[Z_{\lambda}-\lambda f_{i}\left(\theta_{i}\right)\right] \\
& \geq a_{i}\left[Z_{\lambda}-\lambda f_{i}\left(H_{\lambda}\right)\right]>0, \quad t \in(0,1)
\end{aligned}
$$

by the choice of $Z_{\lambda}$. Note that $\left(Z_{\lambda} \varphi_{i}-\theta_{i}\right)(0)=0$. Now we show that $\left(Z_{\lambda} \varphi_{i}-\theta_{i}\right)(1) \geq 0$. Indeed, since $Z_{\lambda} \varphi_{i}^{\prime}(1)+c_{i}\left(Z_{\lambda}\left(\varphi_{i}(1)\right)\right) Z_{\lambda} \varphi_{i}(1) \geq 0=\theta_{i}^{\prime}(1)+c_{i}\left(\theta_{i}(1)\right) \theta_{i}(1)$, we have

$$
Z_{\lambda} \varphi_{i}^{\prime}(1)-\theta_{i}^{\prime}(1)+c_{i}\left(Z_{\lambda}\left(\varphi_{i}(1)\right)\right) Z_{\lambda} \varphi_{i}(1)-c_{i}\left(\theta_{i}(1)\right) \theta_{i}(1) \geq 0
$$

If we assume that $Z_{\lambda} \varphi_{i}(1)-\theta_{i}(1)<0$, then $c_{i}\left(Z_{\lambda}\left(\varphi_{i}(1)\right)\right) Z_{\lambda} \varphi_{i}(1)-c_{i}\left(\theta_{i}(1)\right) \theta_{i}(1)<0$ since $c_{i}$ increases. Hence from (3.3) we obtain $Z_{\lambda} \varphi_{i}^{\prime}(1)-\theta_{i}^{\prime}(1)>0$. However, $-\left(Z_{\lambda} \varphi_{i}-\theta_{i}\right)^{\prime \prime}>0$ in $(0,1)$, $\left(Z_{\lambda} \varphi_{i}-\theta_{i}\right)(0)=0$ and $\left(Z_{\lambda} \varphi_{i}-\theta_{i}\right)(1)<0$ implies that $\left(Z_{\lambda} \varphi_{i}-\theta_{i}\right)^{\prime}(1)>0$, which is a contradiction. Hence $Z_{\lambda} \varphi_{i}(1)-\theta_{i}(1) \geq 0$. Therefore, by the maximum principle, $Z_{\lambda} \varphi_{i} \geq \varphi_{i}$ in $[0,1]$. Therefore, (1.3) has a maximal positive solutions $\tilde{u}, \tilde{v}$. Now, let $u$ and $v$ be any other positive solutions of (1.3). To establish our theorem, we will show that $u \equiv \tilde{u}$ and $v \equiv \tilde{v}$ for $\lambda \gg 1$. Since $u, v$ and $\tilde{u}, \tilde{v}$ are solutions of (1.3), we obtain

$$
\left\{\begin{array}{l}
-(\tilde{u}-u)^{\prime \prime}(t)=\lambda a_{1}(t)\left(f_{1}(\tilde{u}(t))-f_{1}(u(t))\right), \quad t \in(0,1) \\
-(\tilde{v}-v)^{\prime \prime}(t)=\lambda a_{2}(t)\left(f_{2}(\tilde{v}(t))-f_{2}(v(t))\right), \quad t \in(0,1) \\
(\tilde{u}-u)(0)=(\tilde{v}-v)(0)=0, \\
(\tilde{u}-u)^{\prime}(1)+c_{1}(\tilde{u}(1)) \tilde{u}(1)-c_{1}(u(1)) u(1)=0, \\
(\tilde{v}-v)^{\prime}(1)+c_{2}(\tilde{v}(1)) \tilde{v}(1)-c_{2}(v(1)) v(1)=0
\end{array}\right.
$$


By the mean value theorem, there exists $\xi$ such that $u \leq \xi \leq \tilde{u}$ and $v \leq \xi \leq \tilde{v}$ quadin $[0,1]$ and

$$
\left\{\begin{array}{l}
-(\tilde{u}-u)^{\prime \prime}(t)=\lambda a_{1}(t) f_{1}^{\prime}(\xi)(\tilde{u}(t)-u(t)), \quad t \in(0,1) \\
-(\tilde{v}-v)^{\prime \prime}(t)=\lambda a_{2}(t) f_{2}^{\prime}(\xi)(\tilde{v}(t)-v(t)), \quad t \in(0,1) \\
(\tilde{u}-u)(0)=(\tilde{v}-v)(0)=0, \\
(\tilde{u}-u)^{\prime}(1)+c_{1}(\tilde{u}(1)) \tilde{u}(1)-c_{1}(u(1)) u(1)=0, \\
(\tilde{v}-v)^{\prime}(1)+c_{2}(\tilde{v}(1)) \tilde{v}(1)-c_{2}(v(1)) v(1)=0
\end{array}\right.
$$

By multiplying (1.3) by $(\tilde{u}-u),(\tilde{v}-v)$ and (1.32) by $u, v$ and integrating, we first obtain, using integration by parts,

$$
\begin{aligned}
\int_{0}^{1}\left[(\tilde{u}-u)^{\prime \prime} u-(\tilde{u}-u) u^{\prime \prime}\right] \mathrm{d} t & =\tilde{u}^{\prime}(1) u(1)-u^{\prime}(1) \tilde{u}(1) \\
& =u(1)\left[-c_{1}(\tilde{u}(1)) \tilde{u}(1)\right]+\tilde{u}(1)\left[c_{1}(u(1)) u(1)\right] \\
& =u(1) \tilde{u}(1)\left[c_{1}(u(1))-c_{1}(\tilde{u}(1))\right] \\
& \leq 0 \\
\int_{0}^{1}\left[(\tilde{v}-v)^{\prime \prime} v-(\tilde{v}-v) v^{\prime \prime}\right] \mathrm{d} t & =\tilde{v}^{\prime}(1) v(1)-v^{\prime}(1) \tilde{v}(1) \\
& =v(1)\left[-c_{2}(\tilde{v}(1)) \tilde{v}(1)\right]+\tilde{v}(1)\left[c_{2}(v(1)) v(1)\right] \\
& =v(1) \tilde{v}(1)\left[c_{2}(v(1))-c_{2}(\tilde{v}(1))\right] \\
& \leq 0
\end{aligned}
$$

since $c_{i}$ are increasing. Using that $f_{i}$ is concave, we also have

$$
\begin{aligned}
& \int_{0}^{1}\left[(\tilde{u}-u)^{\prime \prime} u-(\tilde{u}-u) u^{\prime \prime}\right] \mathrm{d} t=\lambda \int_{0}^{1} a_{1}(t)\left[f_{1}(u)-f_{1}^{\prime}(\xi) u\right](\tilde{u}-u) \mathrm{d} t \\
& \geq \lambda \int_{0}^{1} a_{1}(t)\left[f_{1}(u)-f_{1}^{\prime}(u) u\right](\tilde{u}-u) \mathrm{d} t \\
& \int_{0}^{1}\left[(\tilde{v}-v)^{\prime \prime} v-(\tilde{v}-v) v^{\prime \prime}\right] \mathrm{d} t=\lambda \int_{0}^{1} a_{2}(t)\left[f_{2}(v)-f_{2}^{\prime}(\xi) v\right](\tilde{v}-v) \mathrm{d} t \\
& \geq \lambda \int_{0}^{1} a_{2}(t)\left[f_{2}(v)-f_{2}^{\prime}(v) v\right](\tilde{v}-v) \mathrm{d} t
\end{aligned}
$$

From (F1), there exist $r_{i}>0, b_{i}>0$ such that $f_{i}(s)-f^{\prime}(s) s \geq b_{i}$ whenever $s \geq r_{i}$. From (1.20), for $\lambda \gg 1, u(t) \geq r_{i}$ and $v(t) \geq r_{i}$ if $d(t, \partial \Omega) \geq \frac{r_{i}}{\lambda C}$. Let $\Omega_{+}=\left[\frac{r_{i}}{\lambda C}, 1-\frac{r_{i}}{\lambda C}\right]$ and $\Omega_{-}=\left(0, \frac{r_{i}}{\lambda C}\right) \cup\left(1-\frac{r_{i}}{\lambda C}, 1\right)$. Then from (1.33), we have

$$
\begin{gathered}
0 \geq \lambda \int_{\Omega_{+}} a_{1}(t) b_{1}(\tilde{u}-u) \mathrm{d} t+\lambda \int_{\Omega_{-}} a_{1}(t) f_{1}(0)(\tilde{u}-u) \mathrm{d} t \\
0 \geq \lambda \int_{\Omega_{+}} a_{2}(t) b_{2}(\tilde{v}-v) \mathrm{d} t+\lambda \int_{\Omega_{-}} a_{2}(t) f_{2}(0)(\tilde{v}-v) \mathrm{d} t .
\end{gathered}
$$

since when $f_{i}$ is concave $f_{i}(W)-W f_{i}^{\prime}(W) \geq f_{i}(0)$ for all $W \geq 0$.

Next let $m$ and $h$ satisfy

$$
-m^{\prime \prime}(t)=\omega_{\Omega_{+}} a_{1}(t), \quad t \in(0,1), \quad m(0)=m(1)=0,
$$




$$
m^{\prime \prime}(t)=\omega_{\Omega_{+}} a_{2}(t), \quad t \in(0,1), \quad m(0)=m(1)=0
$$

and

$$
\begin{array}{ll}
-h^{\prime \prime}(t)=\omega_{\Omega_{-}} a_{1}(t), \quad t \in(0,1), \quad h(0)=h(1)=0, \\
-h^{\prime \prime}(t)=\omega_{\Omega_{-}} a_{2}(t), \quad t \in(0,1), \quad h(0)=h(1)=0
\end{array}
$$

respectively. Now multiplying (1.32) by $b_{i} m+f_{i}(0) h$ and integrating, we obtain

$$
\begin{aligned}
I:= & \int_{0}^{1}-(\tilde{u}-u)^{\prime \prime}\left[b_{1} m+f_{1}(0) h\right] \mathrm{d} t \\
J:= & \int_{0}^{1}-(\tilde{v}-v)^{\prime \prime}\left[b_{2} m+f_{2}(0) h\right] \mathrm{d} t \\
= & (\tilde{u}(1)-u(1))\left[b_{1} m^{\prime}(1)+f_{1}(0) h^{\prime}(1)\right]+\int_{\Omega_{+}} a_{1}(t) b_{1}(\tilde{u}-u) \mathrm{d} t \\
& +\int_{\Omega_{-}} a_{1}(t) f_{1}(0)(\tilde{u}-u) \mathrm{d} t \\
= & (\tilde{v}(1)-v(1))\left[b_{2} m^{\prime}(1)+f_{2}(0) h^{\prime}(1)\right]+\int_{\Omega_{+}} a_{2}(t) b_{2}(\tilde{v}-v) \mathrm{d} t \\
& +\int_{\Omega_{-}} a_{2}(t) f_{2}(0)(\tilde{v}-v) \mathrm{d} t \\
& \left\{\begin{array}{l}
=I_{1}+I_{2} \\
=
\end{array} J_{1}+J_{2}\right.
\end{aligned}
$$

Note that as $\lambda \rightarrow \infty, m \rightarrow e_{i}$ and $h \rightarrow 0$ in $C^{1}[0,1]$. Hence, for $\lambda$ large, we obtain $b_{i} m+f_{i}(0) h>0$, in $(0,1)$ and $i=1,2$

$$
b_{i} m+f_{i}(0) h>0 \text { in }(0,1)
$$

and

$$
b_{i} m^{\prime}+f_{i}(0) h^{\prime}<0
$$

Hence for $\lambda \gg 1,(1.37)$ implies $I_{1} \leq 0, \quad J_{1} \leq 0$ and combining with (1.34) (which implies $I_{2} \leq 0$ and $J_{2} \leq 0$ ) we have $I \leq 0$ and $J \leq 0$. However, by (1.32), we also have

$$
\begin{aligned}
I:= & \int_{0}^{1}-(\tilde{u}-u)^{\prime \prime}\left[b_{1} m+f_{1}(0) h\right] \mathrm{d} t \\
& +\lambda \int_{0}^{1} a_{1}(t) f_{1}^{\prime}(\xi)(\tilde{u}(t)-u(t))\left[b_{1} m+f_{1}(0) h\right] \mathrm{d} t \\
J:= & \int_{0}^{1}-(\tilde{v}-v)^{\prime \prime}\left[b_{2} m+f_{2}(0) h\right] \mathrm{d} t \\
& +\lambda \int_{0}^{1} a_{2}(t) f_{2}^{\prime}(\xi)(\tilde{v}(t)-v(t))\left[b_{2} m+f_{2}(0) h\right] \mathrm{d} t
\end{aligned}
$$

Now for $\lambda \gg 1$, using (1.36), $a_{i}>0$, and $f_{i}^{\prime} \geq 0$ we get $I \geq 0$ and $J \geq 0$. Hence, we conclude that $I \equiv 0$ and $J \equiv 0$ for $\lambda \gg 1$, which implies that $\tilde{v} \equiv v$ and $\tilde{u} \equiv u$ in $[0,1]$. This proves that (1.3) has a unique positive solution for all $\lambda$ large.

\section{Conclusion}

In the paper, were studied the positive radial solutions for elliptic systems to the 
nonlinear Boundary Value problems. And then, we presented that by the Theorem 1.1, and Theorem 2.2, we can obtain a solution of the problem (1.3). Moreover, for all $\lambda \gg 1$, then (1.3) has a unique positive solution.

\section{Acknowledgements}

The authors would like to thank the anonymous referees for their helpful comments.

\section{Conflicts of Interest}

The authors declare no conflicts of interest regarding the publication of this paper.

\section{References}

[1] Berestycki, H., Caffarelli, L.A. and Nirenberg, L. (1996) Inequalities for SecondOrder Elliptic Equations with Applications to Unbounded Domains, I. Duke Mathematical Journal, 81, 467-494.

[2] Lions, P.L. (1982) On the Existence of Positive Solutions of Semilinear Elliptic Equations. SIAM Review, 24, 441-467. https://doi.org/10.1137/1024101

[3] Ali, J., Castro, A. and Shivaji, R. (1993) Uniqueness and Stability of Nonnegative Solutions for Semipositone Problems in a Ball. Proceedings of the American Mathematical Society, 117, 775-782. https://doi.org/10.1090/S0002-9939-1993-1116249-5

[4] Anuradha, V., Hai, D.D. and Shivaji, R. (1996) Existence Results for Superlinear Semipositone Boundary Value Problems. Proceedings of the American Mathematical Society, 124, 757-763. https://doi.org/10.1090/S0002-9939-96-03256-X

[5] Castro, A., Gadam, S. and Shivaji, R. (1997) Positive Solution Curves of Semipositone Problems with Concave Nonlinearities. Proceedings of the Royal Society of Edinburgh Section A: Mathematics, 127, 921-934. https://doi.org/10.1017/S0308210500026809

[6] Castro, A. and Shivaji, R. (1989) Nonnegative Solutions for a Class of Radially Symmetric Nonpositone Problems. Proceedings of the American Mathematical Society, 106, 735-740. https://doi.org/10.1090/S0002-9939-1989-0949875-3

[7] Dancer, E.N. and Shi, J. (2006) Uniqueness and Nonexistence of Positive Solutions to Semipositone Problems. Bulletin of the London Mathematical Society, 38, 1033 1044. https://doi.org/10.1112/S0024609306018984

[8] Oruganti, S., Shi, J. and Shivaji, R. (2002) Diffusive Logistic Equation with Constant Yield Harvesting, I: Steady States. Transactions of the American Mathematical Society, 354, 3601-3619. https://doi.org/10.1090/S0002-9947-02-03005-2

[9] Castro, A., Sankar, L. and Shivaji, R. (2012) Uniqueness of Nonnegative Solutions for Semipositone Problems on Exterior Domains. Journal of Mathematical Analysis and Applications, 394, 432-437. https://doi.org/10.1016/j.jmaa.2012.04.005

[10] Ko, E., Lee, E. and Shivaji, R. (2013) Multiplicity Results for Classes of Singular Problems on an Exterior Domain. Discrete and Continuous Dynamical Systems, 33, 5153-5166. https://doi.org/10.3934/dcds.2013.33.5153

[11] Sankar, L., Sasi, S. and Shivaji, R. (2013) Semipositone Problems with Falling Zeros on Exterior Domains. Journal of Mathematical Analysis and Applications, 401, 146 153. https://doi.org/10.1016/j.jmaa.2012.11.031 
[12] Gordon, P.V., Ko, E. and Shivaji, R. (2014) Multiplicity and Uniqueness of Positive Solutions for Elliptic Equations with Nonlinear Boundary Conditions Arising in a Theory of Thermal Explosion. Nonlinear Analysis. Real World Applications, 15, 51-57. https://doi.org/10.1016/j.nonrwa.2013.05.005

[13] Bulter, D., Ko, E., Lee, E. and Shivaji, R. (2014) Positive Radial Solutions for Elliptic Equations on Exterior Domains with Nonlinear Boundary Conditions. Communications on Pure and Applied Analysis, 13, 2713-2731.

https://doi.org/10.3934/cpaa.2014.13.2713

[14] Castro, A., Hassanpour, M. and Shivaji, R. (1995) Uniqueness of Non-Negative Solutions for a Semipositone Problem with Concave Nonlinearity. Communications in Partial Differential Equations, 20, 1927-1936.

https://doi.org/10.1080/03605309508821157

[15] Ambrosetti, A., Arcoya, D. and Buffoni, B. (1994) Positive Solutions for Some SemiPositone Problems via Bifurcation Theory. Differential Integral Equations, 7, 655 663. https://projecteuclid.org/euclid.die/1370267698 